\title{
Idéologie égalitaire et libération des habitants du ciel dans la série Valérian et Laureline
}

\section{Sylvain Rheault, Université de Regina}

La série de bande dessinée Valérian et Laureline de Pierre Christin et Jean-Claude Mézières, aujourd'hui considérée comme achevée, compte vingt-deux albums parus entre 1967 et 2013, sans compter quelques parutions hors-collection. Comme Luc Besson en a sorti une version cinématographique le 21 juillet 2017, avec un budget de cent-soixante-dix-huit millions d'euros, il semble opportun de redécouvrir cette série qui a fortement influencé la science-fiction, non seulement en France, mais aussi dans le monde.

Dans cet article, certains des choix narratifs de la série complète seront analysés en utilisant une grille structurée autour du concept d'interaction, soit le plus petit élément constitutif d'une narration. Par exemple, dans le combat de David contre Goliath, l'interaction principale suivante serait la suivante : «David transforme 1'humain vivant Goliath en cadavre ». On constate tout de suite que cet élément narratif combine en fait deux « entités » (David, Goliath) et une action (tuer). L'une des entités agit sur l'autre et cette dernière subit une transformation. Tvetan Todorov considère dans Les genres du discours que les unités s'organisent dans un rapport de succession et de transformation (66). C'est parce qu'une transformation a eu lieu que la narration progresse. Dans le contexte du voyage dans le temps, la notion de succession est particulièrement intéressante, mais il faudra pour l'instant la laisser de côté, quitte à en parler dans un autre article. Thierry Groensteen, dans Système de la bande dessinée, récupère le concept de transformation de Todorov pour élaborer ce qu'il appelle l'arthrologie, soit « des relations pouvant s'établir entre les images » (27). En faisant abstraction de la variété des entités que des actions, en fin de compte il n'existe qu'un nombre limité de types de transformations: transformation de la substance, de l'identité ou du statut (voir le tableau des interactions élémentaires à la fin de cet article). Dans l'exemple vu précédemment, c'est sur la substance même de Goliath que David a agi, en transformant un corps vivant en cadavre. Dans le tableau, il s'agit de l'interaction de meurtre. Dans la même catégorie de transformation se trouvent les interactions du voyage, du travail, de la métamorphose et de l'apparition. Viennent ensuite les transformations de l'identité (soi, l'autre) d'une autre entité, comme dans l'acquisition, le troc, la 
communication ou l'union, puis les transformations de statut (chose, personne, idole), comme dans les interactions d'idolisation, de destitution, de personnification ou de chosification. À partir de ce petit nombre d'interactions élémentaires, en permutant les identités, en substituant les entités initiales pour d'autres ou en utilisant une action différente, on peut obtenir un nombre astronomique de variantes.

Afin d'homogénéiser la représentation des interactions, il a fallu établir une constante, soit un humain ayant le statut de «personne » dans l'identité de « soi » et jouant le rôle d'agent, donc qui sera à l'origine de l'action transformant « l'autre ». Pour simplifier, on donnera à cette constante le nom de «héros », mais dans le sens strict d'actant narratif. Dans le tableau, les regroupements d'interactions s'organisent en fonction du statut de «l'autre». Ainsi, dans un premier groupe d'interactions, le héros interagit avec des entités ayant le statut de «chose », principalement avec des objets. Dans le second groupe, le héros interagit avec des entités ayant le statut de «personne », principalement avec d'autres humains (ou leur équivalent extra-terrestre dans la série Valérian et Laureline). Enfin, dans le dernier groupe, le héros interagit avec une autre entité afin de transformer le statut de cette dernière. Parfois, des interactions transformant le statut de «l'autre» se combinent avec d'autres interactions élémentaires, pour former des interactions hybrides. Chacune des interactions du tableau sera décrite succinctement au moment d'en parler, afin de mieux en comprendre les rouages et les implications. L'analyse de la série Valérian et Laureline, devrait permettre de dégager la structure narrative de la série tout en mettant en évidence les tendances évolutives, s'il y en a. Avant de structurer les interactions, il faut établir l'idéologie identitaire de la série afin de déterminer si les différentes interactions relevées s'opposent à cette idéologie ou servent à l'établir.

\section{L'idéologie identitaire}

L'idéologie identitaire articule les choix narratifs des créateurs, en établissant une hiérarchie entre les entités et en priorisant les actions choisies par le héros pour effectuer des transformations. En fait, la narration participe à illustrer et à valider l'idéologie identitaire adoptée par les créateurs. La figure 4 permet de mieux comprendre la structuration des entités significatives dans la série Valérian et Laureline. La case «1» («soi » ayant le statut de «personne ») contient non pas un mais deux humains, Valérian et Laureline, qui représentent ici 
la constante du «héros ». Généralement, le lecteur adopte l'idéologie identitaire proposée, selon le principe bien connu de l'identification avec le héros (voir entre autres Identification with Media Characters de Jonathan Cohen), à moins que cette idéologie ne vienne heurter ses convictions propres. Dans la case «2» («l'autre » ayant le statut de «personne ») se rangeront les humains et les extra-terrestres, que les auteurs désignent comme des « habitants du ciel » dans un album hors-série qui porte justement ce titre. Les autres humains de même que les extraterrestres bénéficient d'un statut égal en tout point à celui de nos héros. Malgré des différences physionomiques parfois énormes, l'ordre naturel au sein de l'univers de la série se fonde clairement sur l'égalité entre toutes les formes de vie intelligentes, c'est-à-dire que tous les individus de la galaxie ont le statut de "personne». Parmi toutes les créatures fantastiques imaginées par les auteurs, celles qui ont une apparence plutôt « humanoïde » bénéficient du statut de «personne» et se distinguent de celles qui présentent des différences morphologiques extrêmes et qui se verront accoler l'étiquette de «monstres ». Les monstres occupent la case « 4 » ( «l'autre » ayant le statut de «chose») et adhèrent à une idéologie identitaire différente de celle des héros. En effet, le monstre s'arroge le statut de «personne» ou «d'idole» et range les habitants du ciel dans le statut de «chose ». Certains monstres se reconnaissent aussi par le fait qu'ils n'ont pas la capacité de communiquer au moyen du langage. Quant au cadavre, il symbolise habituellement la chosification du vivant et constitue l'entité intrinsèque de l'interaction du meurtre. La case «3» («soi » ayant le statut de «chose») représente les possessions de Valérian et Laureline, dont leur vaisseau spatial ainsi que tous les gadgets technologiques qu'ils utilisent pour compléter leurs missions. Enfin, les cases «5» et « 6 » devraient normalement rester vides, puisque dans l'univers de la série, personne n'a le droit de s'approprier un statut supérieur à celui des autres. Ne prétendront à ce statut que les monstres, et il faudra alors que nos héros les en fassent tomber.

\section{Interactions s'opposant à l'idéologie identitaire}

\section{L'asservissement, ou la chosification des habitants du ciel}

Les agents de l'asservissement, de l'acquisition illégitime et du meurtre seront plutôt les «antagonistes » de Laureline et Valérian que ces derniers devront éventuellement éliminer ou chasser. L'asservissement se définit comme un type de chosification, c'est-à-dire une interaction 
où le héros transforme le statut de «personne » d'une entité en statut de « chose ». Réduire un humain à l'esclavage constitue une forme de chosification et ce sont les tyrans, comme la gigantesque créature gélatineuse dans Les oiseaux du maître qui parquent les habitants du ciel dans l'asservissement et s'efforcent de les maintenir les. Gérard Klein remarque d'ailleurs que «[Valérian et Laureline] débarquent presque toujours sur un monde clos ou prisonnier» (en ligne). D'ailleurs, les distinctions de statut au sein des peuples de l'espace apparaissent toujours suspectes aux yeux de Valérian et Laureline et c'est contre ce mal que ceux-ci luttent le plus fréquemment. La figure 5, tirée de l'album L'empire des mille planètes, montre un exemple de chosification extrême. Valérian, enfermé dans un bloc solide, est littéralement devenu un objet, une chose.

Valérian lui-même n'est pas un adepte parfait de sa propre idéologie identitaire puisqu'il s'adonne à l'esclavage en capturant ou trompant des habitants du ciel afin de les vendre à un seigneur de la guerre dans l'album Les armes vivantes. Laureline, pour sa part, n'a jamais une seule fois failli à son idéologie identitaire. De fait, l'amour de Valérian pour Laureline incitera le héros à libérer ceux qu'il venait d'asservir pour collaborer avec eux. Dans le même album (v.5 ; 125 ) et dans d'autres, dont $A u$ bord $d u$ grand rien $(\mathrm{v} .7 ; 25)$, Valérian se montre plutôt brutal envers le Schniarfeur, une créature au tempérament violent qui attaque à coup de jets d'acide enflammés presque tout ce qui bouge. Cependant, le comportement du Schniarfeur change complètement quand on lui «ligature la glande chabounale », une opération similaire au fait d'enclencher le cran de sureté d'une arme à feu. Curieusement, le Schniarfeur cherche constamment la domination d'un maître et c'est probablement cet asservissement volontaire qui lui vaut l'antipathie de Valérian. Le Schniarfeur s'auto-chosifie, en quelque sorte.

\section{L'auto-idolisation du tyran}

Le tyran camoufle l'asservissement des autres sous une apparente élévation de son statut. En effet, si dans l'interaction d'idolisation élémentaire le héros élève une autre entité au statut « d'idole » en permutant les rôles, on obtient la variante de l'auto-idolisation, soit quand un tyran proclame unilatéralement que son statut sera dorénavant supérieur à celui des autres. En réalité, ce mouvement vers le haut cache plutôt un mouvement vers le bas pour les habitants du ciel, puisque ceux-ci perdent tout ou partie de leurs droits. D'autre part, dans un univers où tous les «humanoïdes » sont perçus comme égaux, le statut «d'idole» ne peut s'obtenir légitimement 
que s'il est alloué par les pairs. S'arroger soi-même le statut «d'idole» constitue une grave infraction à l'idéologie identitaire.

\section{L'acquisition illégitime}

Dans l'interaction de l'acquisition, le héros transforme la propriété d'une entité «autre » ayant le statut de «chose» en faisant passer celle-ci dans le domaine des « choses » à «soi ». Dans le corpus, l'exemple le plus significatif se trouve sans aucun doute dans l'albun Bienvenue sur Alflolol, alors que les terriens colonisent sans vergogne la planète Technorog afin d'en exploiter les vastes ressources, qu'on dit inépuisables (v.2; 69). Cette acquisition incontrôlée se voit soudainement remise en question lorsque les habitants légitimes, dont l'espérance de vie s'étend jusqu'à vingt-cinq mille ans, reviennent après un «petit voyage » de quatre mille ans (v.2;80). Toute cette affaire rappelle sans équivoque la dépossession dont ont été victimes les peuples colonisés de l'histoire humaine, qu'il s'agisse des peuples de la Méditerranée sous la domination des Romains ou des peuples d'Amérique sous la domination des Européens. L'acquisition illégitime des ressources par les Terriens constitue une infraction grave au maintien de l'idéologie identitaire et Laureline prend sans hésiter le parti des Alfloliens contre ses semblables. Valérian, quant à lui, s'efforce maladroitement de trouver un terrain d'entente entre le colonisateur et le peuple d'origine. L'idéologie identitaire du gouverneur s'organise autour des investissements et de la production, qui ont le statut de «personne » à ses yeux, ce qui l'incite à opter pour des interactions rabaissant les Alfloliens au statut de «chose». Pour Valérian et Laureline, tout peuple a droit à son territoire d'origine et ce droit devrait être inviolable. Voler ou coloniser un territoire résulte immanquablement en la chosification du propriétaire légitime, puisque l'un de ses droits fondamentaux, celui de la propriété de son territoire, se voit révoqué.

Valérian kidnappe Ralph sur la planète Glapum't dans l'album Les spectres d'Inverloch et il s'agit d'un cas de chosification. En d'autres mots, Valérian acquiert, ou asservit, une créature intelligente, sans son consentement, comme si elle n'avait que le statut de « chose ». Curieusement, cette action illégitime semble rapidement pardonnée par la victime elle-même, Ralph, qui décide dorénavant d'aider de son plein gré les humains qui le traitent maintenant avec le respect dû aux «personnes » et qui lui offrent des fleurs à manger, incluant de rares orchidées, ainsi que d'autres délicieux mets terriens. Voilà un retournement pour le moins intéressant qui permet d'effacer rapidement le crime de chosification commis par Valérian, à l'encontre de son 
idéologie identitaire.

\section{Le meurtre}

Dans l'interaction du meurtre, le héros transforme un autre humain vivant en cadavre. Du point de vue narratif, le meurtre comporte des résonances dramatiques sérieuses parce que, selon l'idéologie identitaire, il existe une distinction significative entre un humain vivant et son cadavre. En effet, le meurtre, en plus de la transformation en cadavre, implique presque toujours une chosification de «l'autre». Dans la série, un certain nombre de cadavres résultant de meurtres permettent de faire sentir la gravité du meurtre. Dans L'empire des mille planètes, la révolte des peuples opprimés provoque des morts $(\mathrm{v} .1 ; 150)$ et se termine par le suicide collectif (v.1; 153) de tous les Terriens qui voulaient se venger de leur planète d'origine, croyant qu'ils avaient été abandonnés par leurs semblables. Dans Le pays sans étoiles, de pauvres types meurent (v.2; 55) enrôlés malgré eux dans une guerre futile entre deux nations, l'une dirigée par des femmes et l'autre par des hommes. Un humanoïde se fait écraser (v.7 ; 85) par un monolithe géant dans L'ordre des pierres. Ces monolithes de pierre, appelés Woloch, projettent de prendre la place des habitants du ciel au sein de la galaxie. La résistance contre ces monstres impitoyables dans L'ouvretemps occasionnera de nombreuses morts (v.7; 150). L'aspect physique des Woloch, ainsi que leur idéologie identitaire, représentent l'altérité poussée à son degré maximum, puisque ces monstres sont constitués de pierre, ont l'apparence de simples monolithes géants et n'ont aucune considération pour les habitants du ciel. Brefs, ce sont des monstres parfaits.

Si le meurtre des habitants du ciel constitue un crime, en revanche la chosification des monstres va presque de soi. Dans l'album Sur les terres truquées, Valérian et Laureline tentent de retrouver un étrange extra-terrestre qui crée des mondes similaires à la Terre. Afin d'assurer le succès de la mission, des clones de Valérian sont envoyés en mission et finissent par se faire tuer les uns après les autres, de manière extrêmement violente. Visiblement, les clones n'ont que le statut de « chose », d'autant plus que leur durée de vie se limite à quelques heures. On peut donc les sacrifier sans remords, comme des produits de consommation. À l'aune de d'idéologie identitaire, les clones se définissent comme des monstres produits par la science et la gravité du meurtre s'en trouve pour ainsi dire annulée.

Si Valérian et Laureline ne tuent personne, du moins pas de manière directe, il faut noter 
que dans l'album Brooklyn station terminus cosmos, Laureline fait s'entretuer deux petits truands d'origine extra-terrestre, Rackalust et Crocbattles, dont les manigances irréfléchies avaient mis en danger des milliers de personnes sur la planète Terre des années 1980 (v.4; 107). Ostensiblement, Laureline cause leur mort, puisqu'elle les manipule pour qu'ils en viennent à s'entretuer, mais la narration parvient malgré tout à atténuer la gravité de ce meurtre. D'abord, Rackalust et Crocbattles prouvent, par leurs actions, qu'ils sont des « monstres moraux », comme l'étaient Caligula ou Adolf Hitler. Pour des monstres de ce type, les humains ne sont que des entités exploitables et tant pis si certains meurent ou souffrent à cause de leurs actions. Pour ajouter à leur monstruosité, la narration montre que les deux escrocs ne cherchent aucunement à contrôler leurs instincts primaux $(\mathrm{v} .4 ; 110 ; 112)$, ce ne sont que des bêtes. Pour toutes ces raisons, le public ne s'offusque pas de l'exécution sommaire menée par Laureline (voir figure 6) et a même plutôt envie de rire de leur sottise parce qu'ils étaient trop bêtes pour se rendre compte qu'ils allaient se tuer l'un l'autre. D'ailleurs, aucun des monstres n'a droit à des bulles de dialogue. Après la mort des deux escrocs, Laureline détruira sans hésitation les cubes qui faisaient apparaître des monstres sur terre (v.4; 114). En conformité avec l'idéologie identitaire, on peut massacrer les monstres sans remord, comme c'est le cas pour les oiseaux venimeux dans Les oiseaux du maitre $(\mathrm{v} .2 ; 153)$ ou les monstres destinés à éprouver le courage des quatre champions dans Les héros de l'équinoxe (v.3; 141-144).

Le meurtre s'oppose violemment à l'idéologie identitaire, puisque des créatures vivantes ayant le statut de «personne » perdent leur droit de vivre, ce qui constitue une chosification. Par contre, selon cette même idéologie, tuer des monstres reste acceptable et même recommandable. Il faut cependant que la narration indique clairement quelles entités sont à considérer comme des monstres qui, on s'en souvient, n'ont que le statut de «chose» dans l'idéologie identitaire de Valérian et Laureline.

\section{Interactions établissant l'idéologie identitaire}

\section{La libération, ou la personnification des habitants du ciel}

Dans le tableau, l'interaction de la «personnification» prend un sens particulier et doit être envisagée comme un antonyme direct de l'interaction de chosification. En exécutant une personnification, le héros donne ou redonne à des habitants du ciel les droits qui leur assurent le 
plein statut de «personne». Justement, dans L'empire des mille planètes, à l'occasion de leur première mission dans l'espace sidéral, Valérian et Laureline participent à une révolution pour chasser des faux prêtres. Dans l'album L'ambassadeur des ombres, le complot de la terre pour contrôler la planète artificielle Point Central échoue entre autres grâce à l'intervention de Valérian et Laureline et les habitants du ciel conservent ainsi leur indépendance et leur neutralité, donc leur statut de «personne». On note que Valérian et Laureline donnent la priorité à l'idéologie identitaire même si c'est au détriment des visées hégémoniques de leur propre planète d'origine.

Il ne fait aucun doute que la personnification, ou la libération, constitue l'interaction la plus importante des aventures de Valérian et Laureline puisqu'elle établit directement l'idéologie identitaire. C'est l'interaction principale de presque tous les albums de la série, et les autres interactions, qui seront maintenant décrites, sont au service de celle-ci.

\section{La destitution des tyrans}

Avec l'interaction de destitution, le héros rabaisse au statut de «personne » une entité qui s'était précédemment arrogé le statut «d'idole » et qui maintenait sous son joug des humanoïdes

innocents. La destitution, c'est le sort réservé à ceux qui prétendent à un statut supérieur sans y avoir été légitimement appelés. Au sein des albums de la série, la destitution s'exécute directement en opposition à l'auto-idolisation. L'une des missions les plus fréquentes de Valérian et Laureline consiste justement à faire tomber ces faux dieux qui maintiennent des habitants du ciel dans une soumission abjecte. Le cas le plus représentatif de destitution, la gargantuesque monstruosité gélatineuse dans Les oiseaux du maître (v.2; 161), sera chassée dès que les individus du groupe qui l'affronte décident de mettre en commun leurs efforts (voir figure 7). Il y a aussi, dans Les cercles du pouvoir, le démasquage de l'Hyperprince qui se révélera n'être qu'une simple projection holographique (v.5; 201), un symbole d'oppression d'autant plus ironique qu'il n'a aucune substance. Enfin, il y a ces monolithes de pierre (v.7 ; 60) dans les trois derniers albums de la série (Au bord du grand rien, L'ordre des pierres, L'ouvretemps), auquel rien ne semble résister et qui seront finalement terrassés grâce à un tout petit appareil anodin appelé « l'ouvretemps ». 


\section{L'apparition}

Dans cette interaction, le héros transforme une entité «invisible » de manière à la rendre visible. L'invisible ici désigne ce qu'on ne peut pas voir, qu'il s'agisse d'entités spirituelles, donc dépourvues de substance matérielle, ou encore d'entités maintenue cachée du public, comme le coupable dans un roman policier. Lorsqu'il est question de religion dans l'univers de Valérian et Laureline, comme dans L'empire des mille planètes, il s'agit généralement d'une secte bidon dont le but véritable est l'asservissement des habitants du ciel. Dans Les foudres d'Hypsis, Valérian et Laureline révèlent que les membres de la Sainte-Trinité, composée du Père, du Fils et du Saint-Esprit, sont en fait trois entités extra-terrestres en «chair et en os ». Les auteurs ont représenté ceux-ci sous les traits d'un gangster obèse, d'un hippie barbu et d'une machine à sous d'un type ancien, avec un levier. En fait, le Père de la Sainte-Trinité, par son arrogance et sa prétention à un statut «d'idole », est à ranger parmi ceux qui pratiquent l'auto-idolisation, malgré ses traits caricaturaux. Dans l'univers de Valérian, ces trois extra-terrestres étaient «invisibles » en ce sens qu'ils étaient insaisissables. En effet, leur planète, Hypsis, avait la capacité de changer de constellation à son gré $(\mathrm{v} .4 ; 141)$ et était presque impossible à localiser.

C'est aussi au moyen de l'apparition que Valérian et Laureline, dans Les cercles $d u$ pouvoir (v.5 ; 201), démasquent le prince qui se révèle n’être rien de plus qu'un hologramme. Dans ce cas-ci, ainsi que dans les précédents, l'interaction de l'apparition contribue à la destitution et les auteurs en profitent pour tourner la religion en dérision.

\section{La métamorphose}

Dans cette interaction, le héros transforme la substance, c'est-à-dire le corps, d'une autre entité ayant le statut de «personne ». Le héros pourrait aussi envisager de transformer son propre corps et, de fait, l'auto-métamorphose constitue la variante la plus fréquente dans la série puisque l'une des spécialités de Valérian et Laureline consiste justement à transformer leur propre «forme ». En effet, en tant qu'agents spatio-temporels, il est essentiel pour eux de modifier leur apparence afin de se fondre dans les diverses époques de l'histoire humaine ou les diverses civilisations qui fleurissent dans la galaxie. Valérian et Laureline peuvent passer incognito presque partout en se mêlant aux foules, mais c'est aussi une façon de respecter la culture de «l'autre». Comme le remarque Jérôme Dumetz, «Valérian est toujours respectueux des coutumes locales et ne cherche pas à imposer des opinions » (26). D'autre part, certains extra- 
terrestres ont le pouvoir de se métamorphoser, littéralement, comme Brittibrit ou Yfysania dans l'album Les armes vivantes. Le premier peut prendre n'importe quelle forme qui lui est mentalement suggérée, le second peut «posséder » les autres êtres intelligents, et donc prendre la forme de ceux-ci. La principale fonction de la métamorphose consiste à faciliter les interactions de communication, de troc et d'union avec d'autres entités. La métamorphose contribue donc à l'établissement de l'idéologie identitaire d'une manière indirecte mais significative.

\section{Le travail « magique »}

Avec cette interaction, le héros transforme une entité ayant le statut de «chose » pour obtenir un produit différent, que ce soit en forme ou en substance. On parle ici du travail au sens où Marx l'entend, soit un prolongement de l'Homme, ou encore selon le sens de Marshall MacLuhan, pour qui l'activité humaine, incluant le travail, consiste essentiellement en transformation et transmission d'information (25). En quoi consiste exactement le « travail » de Valérian et Laureline ? Officiellement, les deux jeunes gens œuvrent pour le service spatiotemporel qui leur confie des missions. Généralement, cela consiste à se déplacer (voyage), à rencontrer des entités intelligentes (communication), à trouver des alliés (union) et à faire du troc au besoin. Il leur arrive rarement d'utiliser l'interaction du travail, c'est-à-dire qu'ils n'ont pas besoin de transformer la substance d'entités ayant le statut de «chose », comme des produits de consommation. Cependant, pour faire du troc, il faut disposer d'objets à échanger. Dans le monde merveilleux de la science-fiction, presque tout devient possible et, comme par miracle, grâce au transmuteur grognon de Bluxte (v.3, 26 ; v.5, 159), Valérian et Laureline peuvent

littéralement multiplier les petits objets ayant beaucoup de valeur, comme des perles d'ébébé ou des dollars. C'est un travail «magique », c'est-à-dire qu'il suffit de dire, d'utiliser la parole au lieu du travail physique pour obtenir un produit. Il suffit de dire «perles d'ébébé » pour que le petit animal en produise à profusion. Ainsi, l'interaction du «travail magique » procure les ressources nécessaires pour l'interaction du troc et, sans participer directement à l'établissement de l'idéologie identitaire, elle reste cependant indispensable pour que se réalise la libération.

\section{Le voyage}

Le héros, au moyen de l'action «d'aller », transforme le territoire en un autre, le territoire ayant le statut de «chose ». Dans la série, l'entité du territoire se présente sous deux aspects bien distincts : l'espace intersidéral d'une part et le temps d'autre part. Le territoire, et cela devient 
encore plus apparent dans le cas de la science-fiction, doit être conceptualisé comme un espacetemps, c'est-à-dire que le territoire existe non seulement à un endroit donné mais aussi à un moment donné. Le temps, comme entité, se conceptualise donc comme une variante du territoire. Dans l'univers de la série, le cosmos n'est pas aussi vide qu'on pourrait le croire puisque s'y trouvent, entre autres, des astéroïdes ainsi que de nombreuses planètes naturelles ou artificielles où habitent des milliards de créatures de toutes formes. Un humain normalement constitué n'a pas la capacité de se déplacer par ses propres moyens dans l'espace intersidéral et il ne peut non plus y survivre sans équipement sophistiqué. Il faut absolument utiliser des « outils » pour s’y rendre, comme un vaisseau spatial, et enfiler une combinaison spatiale pour y survivre. D'ailleurs, l'uniforme de Valérian et Laureline évoque une combinaison spatiale un peu rétro. La série exploite à fond le voyage dans l'espace intersidéral, l'un des grands thèmes de la sciencefiction. Le temps, envisagé comme un territoire que l'on peut explorer, constitue un autre grand thème unique au genre de la science-fiction. On parle d'ailleurs de ce type d'interaction comme du « voyage dans le temps ». Dans la série Valérian et Laureline, l'interaction avec le temps se conceptualise d'une manière similaire à l'interaction avec l'espace, puisqu'il faut des outils afin d'aller ailleurs dans le temps. Valérian et Laureline disposent d'un vaisseau spatial de type « XB $982 »($ v.1, 115) qui leur permet de faire des sauts à la fois dans le temps et l'espace. Nos deux voyageurs, grâce à la technologie dont ils disposent, peuvent littéralement voyager n'importe où et n'importe quand. Ils peuvent se rendre n'importe où et n'importe quand, ce sont les voyageurs ultimes. Il peut arriver qu'il y ait cooccurrence des deux types de déplacements, comme dans Métro Châtelet direction Cassiopée, alors que Valérian opère au vingtième siècle, sur la Terre, tandis que Laureline enquête au 32e siècle dans une galaxie lointaine.

Klein, déjà cité plus tôt, écrivait : «Ils [Valérian et Laureline] débarquent presque toujours sur un monde clos ou prisonnier. Leur passage - plus que leur action - le rouvre à la diversité cosmique » (en ligne). Le «passage », c'est-à-dire le fait de voyager, accomplit une transformation en soi. Parmi les options offertes pour faire progresser l'interaction identitaire d'une aventure, le voyage permet d'aller là où se trouvent les entités à rencontrer ainsi que l'information à trouver, ou encore les monstres à éliminer. Le voyage participe indirectement, mais de manière indispensable, à l'établissement de l'idéologie identitaire. 


\section{L’union}

Lors d'une interaction d'union, le héros transforme l'identité d'une autre entité de manière à constituer avec celle-ci une nouvelle identité ayant des parties communes avec la sienne. Il peut s'agir de se donner des buts communs, de partager des expériences ou d'établir des liens d'amitié ou d'amour. Dans une perspective métatextuelle, l'union permet de représenter l'identification du lecteur avec les protagonistes en créant, au long du récit, une zone d'expériences, de pensées et d'idéologies communes. Il faut séduire le lecteur afin de le faire adhérer à l'idéologie identitaire qui motive la narration. Au cœur de la série, l'union la plus évidente et la plus forte c'est bien sûr la relation intime entre les deux protagonistes, Valérian et Laureline. Leur union a survécu à de nombreuses crises, incluant des désaccords et des infidélités, et se fonde, d'une part, sur une véritable égalité entre les sexes, les deux partenaires ayant exactement le même statut, et d'autre part sur de nombreuses expériences communes qui ne cessent de s'accumuler tout au long de la série. Des unions se créent aussi avec les habitants $\mathrm{du}$ ciel, surtout quand ces unions participent à l'établissement de l'idéologie identitaire. L'exemple le plus fort se trouve dans Les oiseaux du maître, alors que les sept résistants déterminés à confronter le maître se rendent vite compte que les efforts individuels se font rapidement écrasés mais qu'en unissant les volontés le groupe devient assez forts pour chasser la monstruosité gélatineuse. L'union constitue une interaction indispensable pour parvenir à destituer ceux qui prétendent illégitimement au statut «d'idole». Dans les derniers albums de la série, les enfants de Valérian, qu'il a conçus lors d’une précédente aventure avec la Mère Suprême (v.3; 158) deviendront plus tard, (v.7; 121) des alliés fidèles en vertu de leur lien familial avec leur père. La relation filiale établit une forme d'identité commune qui semble aller de soi. Comme d'autres interactions, l'union, sans participer directement à l'établissement de l'idéologie identitaire, contribue à la destitution des tyrans et des faux dieux.

\section{La communication}

Par le biais de l'interaction de communication, le héros utilise la parole pour transformer les connaissances d'un autre humain en lui transmettant les siennes. On peut donner comme exemple le moment où Laureline surprend Valérian au lit avec une autre femme et s'exclame : «Non, mon petit bonhomme. Ce n'est pas moi qui suis dans le lit à côté de toi » (v.4; 73). On comprend que ce genre d'information peut avoir un impact transformationel décisif, en 
particulier pour la relation du couple. Dans toute la série, il y a énormément de dialogues entre les personnages, comme on peut s'y attendre dans la plupart des bandes dessinées, mais ici l'échange d'information est vital pour résoudre les crises. Il importe de comprendre l'origine de l'oppression et la nature de l'oppresseur avant d'être en mesure de destituer celui-ci.

\section{Le troc}

Le troc représente en fait une variante de la communication où le héros utilise la parole avec une autre entité ayant le statut de «personne», cette fois dans le but de transformer la propriété de deux entités ayant le statut de «chose». La vision du troc, ici, met l'accent sur la relation entre les deux entités ayant le statut de «personne » plutôt que sur l'échange d'objets ayant le statut de «chose ». Lors de leur première mission sur une nouvelle planète, dans l'album L'empire des mille planètes, la toute première interaction de Valérian et Laureline sera l'achat d'une montre dans un marché (v.1; 116), donc à faire du troc. Le troc est une forme de communication, même si son but ultime consiste à échanger des entités ayant le statut de «chose ». Le troc est une manière beaucoup plus respectueuse de rencontrer «l'autre » que l'acquisition.

Dans Les armes vivantes, Valérian se fait trafiquant d'armes vivantes afin de pouvoir réparer son vaisseau. Autrement dit, il fait du troc pour assurer la subsistance du couple. Plus tard, dans l'album Sur les frontières, Valérian et Laureline vendent leurs services aux puissances gouvernementales de la Terre en tant que mercenaires afin, d'une part, de gagner de l'argent, mais aussi dans le but de protéger la terre contre les complots d'extra-terrestres sans scrupules. Habitués au troc, Valérian et Laureline assument le rôle de marchands ambulants aux abords d'une prison dans l'album $A u$ bord du grand rien. Le troc est décidément dans la nature même de nos héros.

Dans la série, l'objet d'échange le plus en demande de la part de Valérian et Laureline, c'est l'information, les auteurs ont justement créé des personnages spécialistes de ce genre de troc : les Shingouz, qu'on rencontre pour la première fois dans L'ambassadeur des ombres (v.3 ; 33). Espions extra-terrestres, ils se déplacent beaucoup et vendent un peu de tout mais en particulier des informations qu'ils glanent ici et là de manière aussi mystérieuse qu'efficace. Dans la figure 8, Laureline négocie avec les Shingouz l'achat d'une carte de Point Central, la planète artificielle. Les participants ont tous le statut de «personne », l'ambiance est détendue et 
on a confiance en la réussite de la négociation. Ce type d'interaction s'oppose diamétralement à l'usage de la violence. Selon Klein, «la circulation concurrentielle qu'ils [les marchands] assurent empêche la concentration du pouvoir et la prise en masse de l'idéologique » (en ligne). Ainsi, le troc se révèle un contrepoids efficace aux différentes formes de pouvoir.

\section{La structure narrative dans Valérian et Laureline}

Dans les analyses qui précèdent, il est apparu que l'interaction principale participe directement à l'établissement de l'idéologie identitaire, tandis que les autres interactions s'organisent soit en appui, soit en opposition à cette interaction principale. Voici une schématisation :

1. L'idéologie identitaire principale :

- Tous les habitants du ciel sont égaux aux humains, sauf s'ils sont des monstres.

2. L'interaction principale :

- La libération des habitants du ciel opprimés (personnification)

3. Interactions s'opposant à l'interaction principale :

- L'asservissement des habitants du ciel (chosification)

- L'acquisition illégitime (acquisition)

- L'utilisation de moyens de coercion (meurtre)

- L'auto-idolisation (idolisation)

4. Interactions contribuant à l'interaction principale :

- A) Faire tomber les fausses idoles (destitution).

- B) Tuer les monstres (meurtre).

- A) Révéler la véritable nature des fausses idoles (apparition).

- B) Trouver où se terre le monstre (apparition).

- Partager de l'information utile (communication).

- Se déplacer pour communiquer (voyage).

- S'adapter aux autres cultures (métamorphose).

- Acheter de l'information utile (troc).

- Produire des objets à échanger (travail).

- Se déplacer pour faire du troc (voyage).

- S'adapter aux autres cultures (métamorphose).

- Établir des alliances (union).

- Se déplacer pour rencontrer habitants du ciel (voyage).

- S'adapter aux autres cultures (métamorphose).

- Se déplacer pour faire tomber l'idole ou tuer le monstre (voyage).

L'idéologie identitaire détermine dès le départ l'interaction principale ainsi que l'interaction qui s'y opposera. La narration évoluera le long de cette tension entre deux interactions antagonistes. On remarque que le troc et la communication sont essentiels aux succès 
des missions. Les échanges d'objets et d'idées permettent à la narration de progresser tout en participant à l'établissement de l'idéologie identitaire. Au début d'une nouvelle aventure, Valérian et Laureline ne disposent pas encore des ressources nécessaires ni des alliés sur lesquels ils pourront compter. Il leur faut ensuite convaincre, acheter, soudoyer, séduire afin d'obtenir l'information qui permettra de passer à l'interaction suivante. Si certaines interactions participent directement à l'établissement de l'idéologie identitaire, d'autres, plus modestes mais tout aussi essentielles, servent plutôt à épauler des interactions. Par exemple, le travail, le voyage et la métamorphose rendent possible le troc et la communication. Le voyage, en particulier, apparaît à deux niveaux. Cela témoigne de son importance dans la trame narrative. Cette structure narrative pourrait être envisagée comme la recette de base de la narration et elle permettrait d'expliquer la plupart des choix narratifs faits au sein de la série dans son entier. On constate cependant que chaque album ajoute ou enlève des ingrédients afin de donner une saveur particulière à l'épisode, mais que dans l'ensemble il s'agit de protéger les droits fondamentaux des habitants du ciel. En fin de compte, cet exercice d'analyse portant sur l'œuvre de Christin et Mézières révèle certainement des particularités narratives intéressantes, mais il faudra analyser un peu plus d'œuvres avant de conclure à l'utilité d'une étude fondée sur les interactions.

\section{Bibliographie - Corpus}

Christin, Pierre et Jean-Claude Mézières. Valérian et Laureline - L'intégrale. Paris : Dargaud.

Volume 1. 2007.

Volume 2. 2008.

Volume 3. 2009.

Volume 4. 2009.

Volume 5. 2011.

Volume 6. 2012.

Volume 7. 2012.

Christin, Pierre et Jean-Claude Mézières. Les habitants du ciel. Paris : Dargaud, 2000.

Titres des albums individuels de la série Valérian et Laureline chez Dargaud :

0. Les mauvais rêves (parution originale : 1967). 2000.

1. La cité des eaux mouvantes. 1970. 
2. L'empire des mille planètes. 1971.

3. Le pays sans étoile. 1972.

4. Bienvenue sur Alflolol. 1972

5. Les oiseaux du maître. 1973.

6. L'ambassadeur des ombres. 1975.

7. Sur les terres truquées. 1977.

8. Les héros de l'équinoxe. 1978.

9. Métro Chatelet direction Cassiopée. 1980.

10. Brooklyn station terminus cosmos. 1981.

11. Les spectres d'Inverloch. 1984.

12. Les foudres d'Hypsis. 1985.

13. Sur les frontières. 1988.

14. Les armes vivantes. 1990.

15. Les cercles du pouvoir. 1994.

16. Otages de l'Ultralum. 1996.

17. L'orphelin des astres. 1998.

18. Par des temps incertains. 2001.

19. Au bord du grand rien. 2004.

20. L'ordre des pierres. 2007.

21. L'ouvretemps. 2010.

22. Souvenirs de futurs. 2013.

\section{Ouvrages cités}

Cohen, Jonathan. Identification with Media Characters. Oxford Bibliographies, 2014. http://www.oxfordbibliographies.com/view/document/obo-9780199756841/obo9780199756841-0144.xml. 19 septembre 2017.

Dumetz, Jérôme. «Valérian : agent interculturel ? - Analyse interculturelle de la bande dessinée culte de science-fiction francophone ». Les cahiers d'études interculturelles 5 (2017).

Groensteen, Thierry. Système de la bande dessinée. Paris : PUF, 1999.

Klein, Gérard. «Des messagers de l'actuel. Une exploration des mondes de Valérian » Mézières 
et Christin avec.... Paris : Dargaud, 1983.

https://www.noosfere.org/mezieres/pages/extras/articles/articleT.asp ?article=valgklein. 18 septembre 2017.

McLuhan, Marshall. Pour comprendre les média (Understanding Media). Les prolongements technologiques de l'homme. Montréal : Hurtubise, 1972 [1964].

Todorov, Tzvetan. Les genres du discours. Paris : Seuil, 1978.

\section{TABLEAU DES INTERACTIONS ÉLÉMENTAIRES}
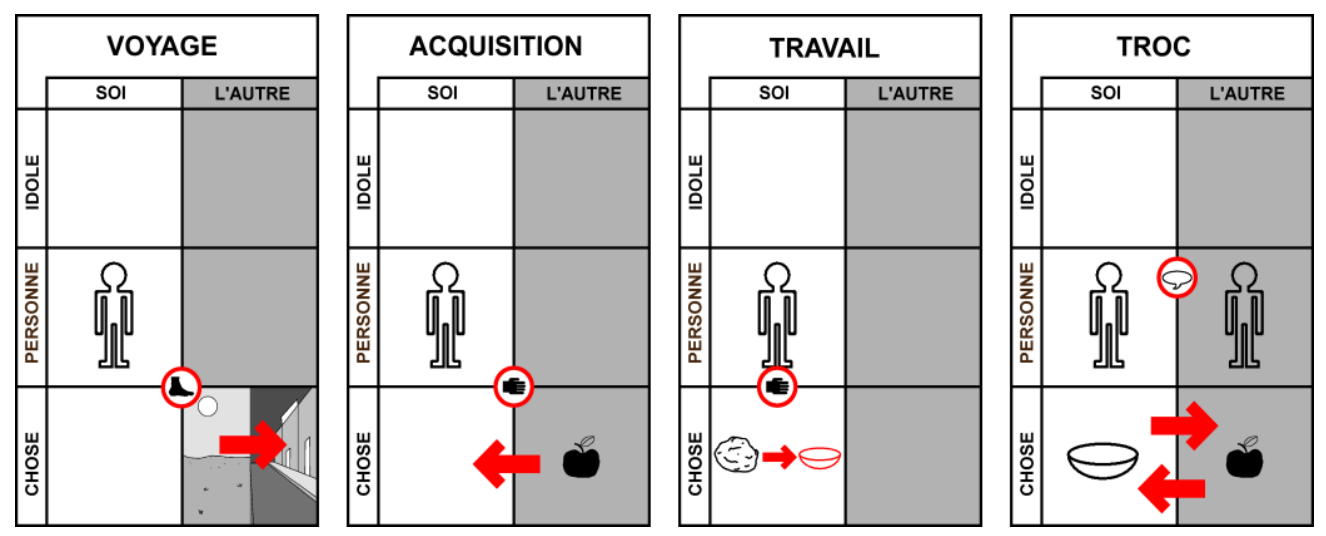

Les interactions élémentaires où « l'autre » a le statut de «chose ».
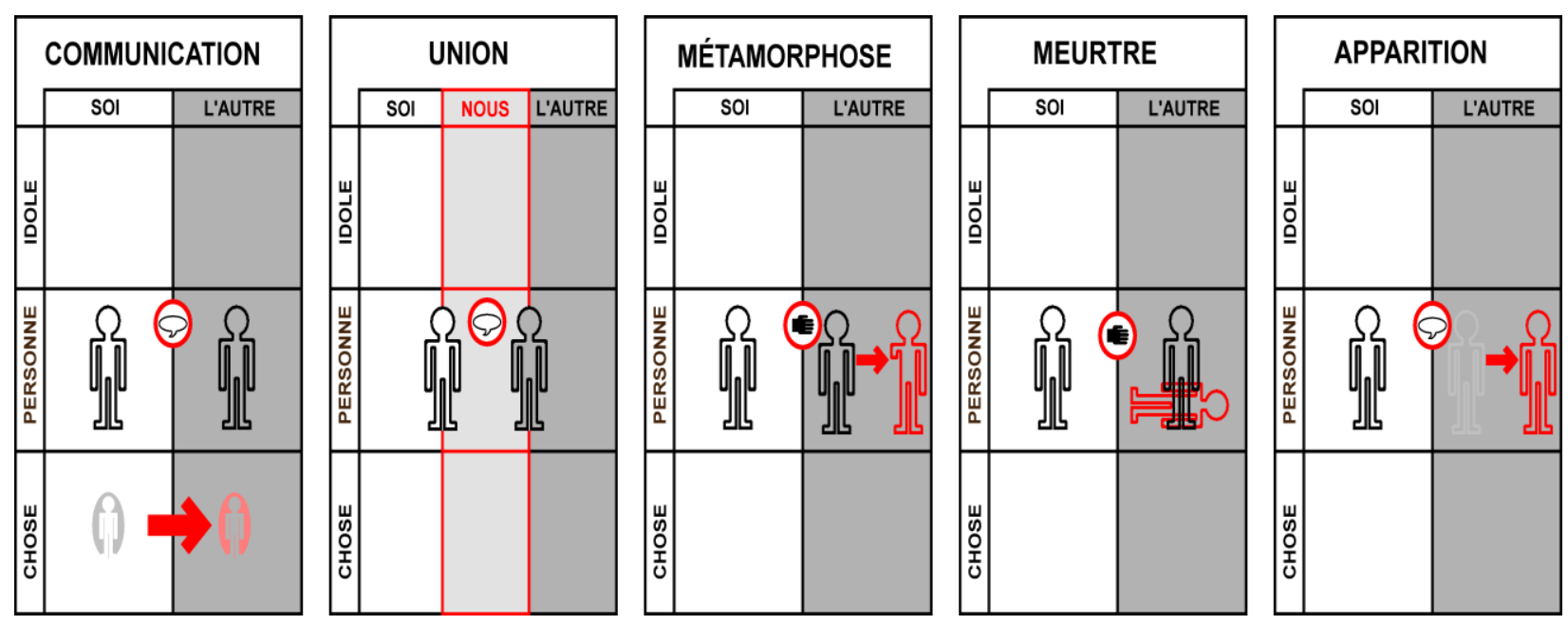

Les interactions élémentaires où « l'autre » a le statut de « personne » 
Les interactions élémentaires transformant le statut de « l'autre »
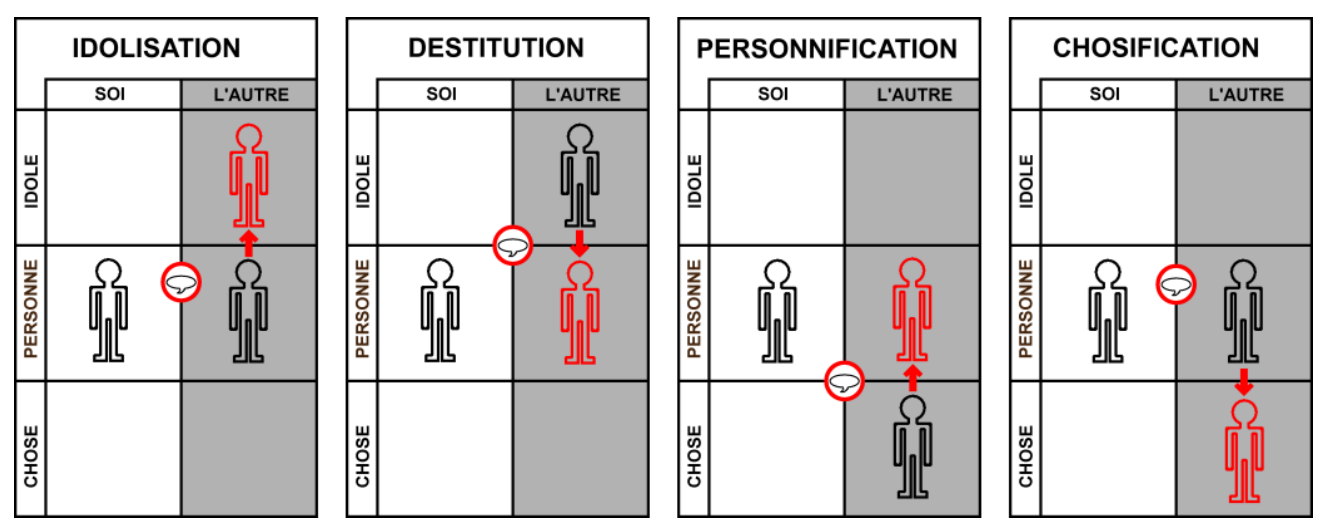


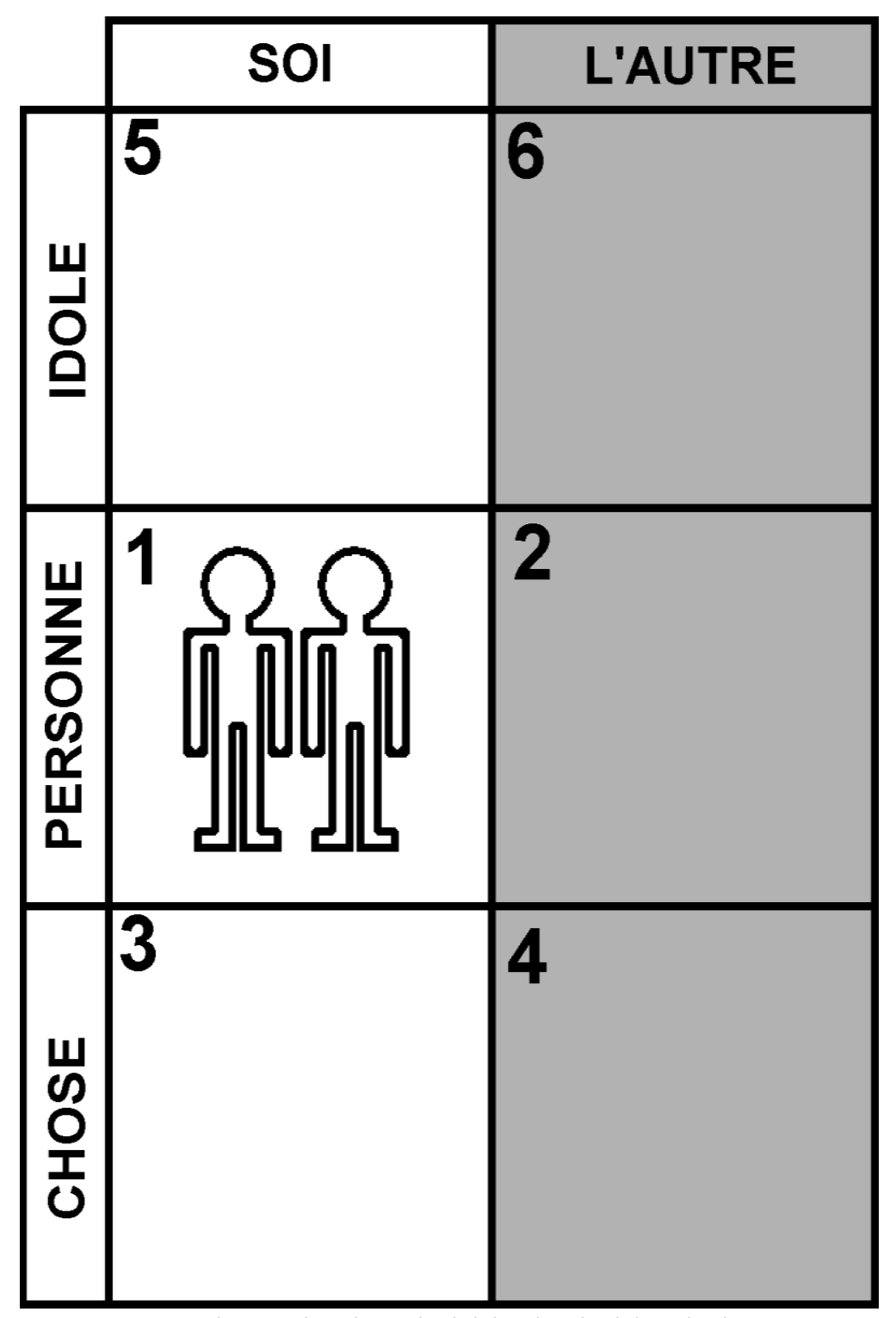

Figure 4 - Schématisation de l'idéologie identitaire. 


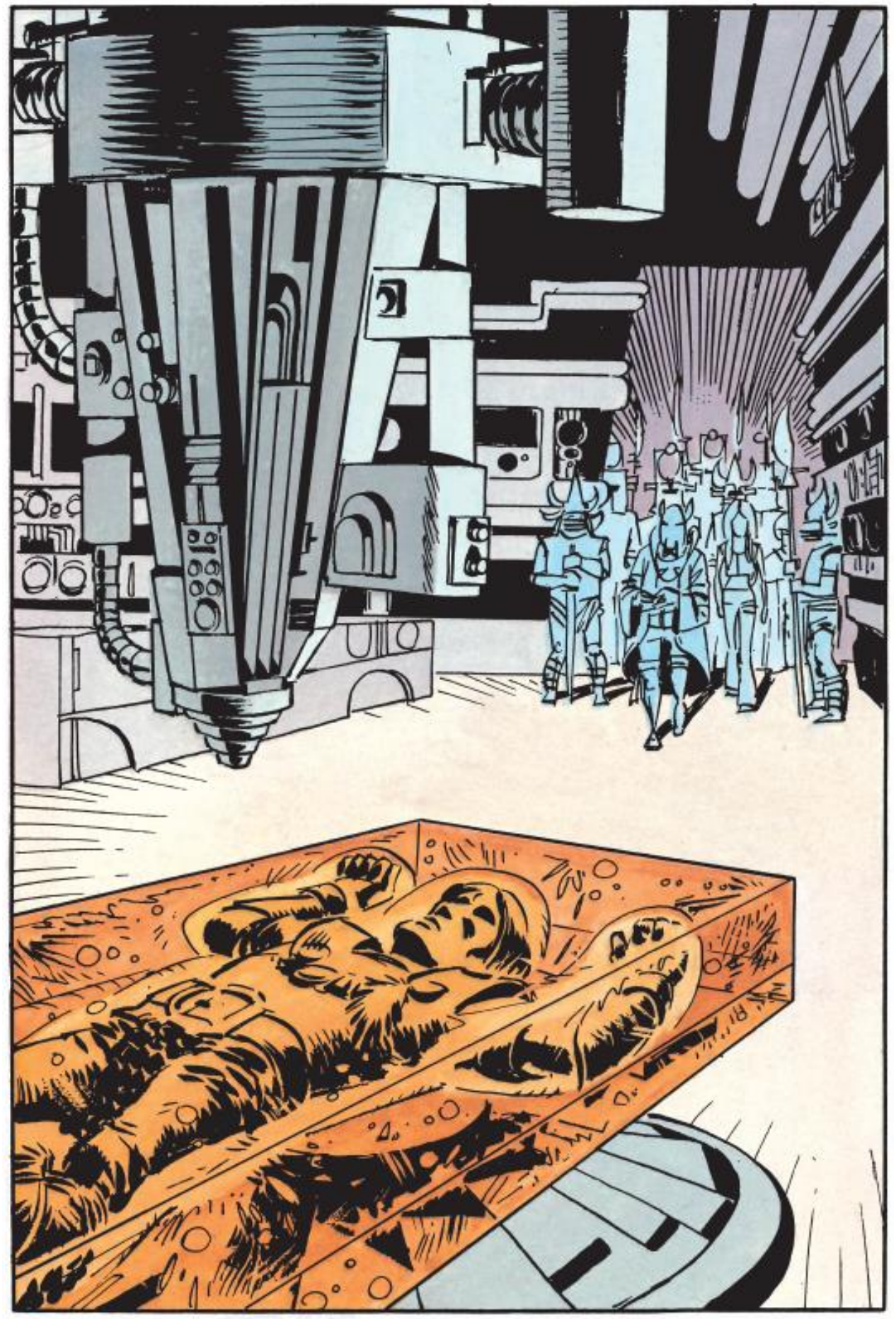

Figure 5 - Exemple de chosification. Enfermé dans son bloc transparent, Valérian semble être devenu une «chose» inerte. Valérian - Intégrale nouvelle édition - volume 1 : case 4 , p. 142, Christin, Mézières (C) DARGAUD, 2017 

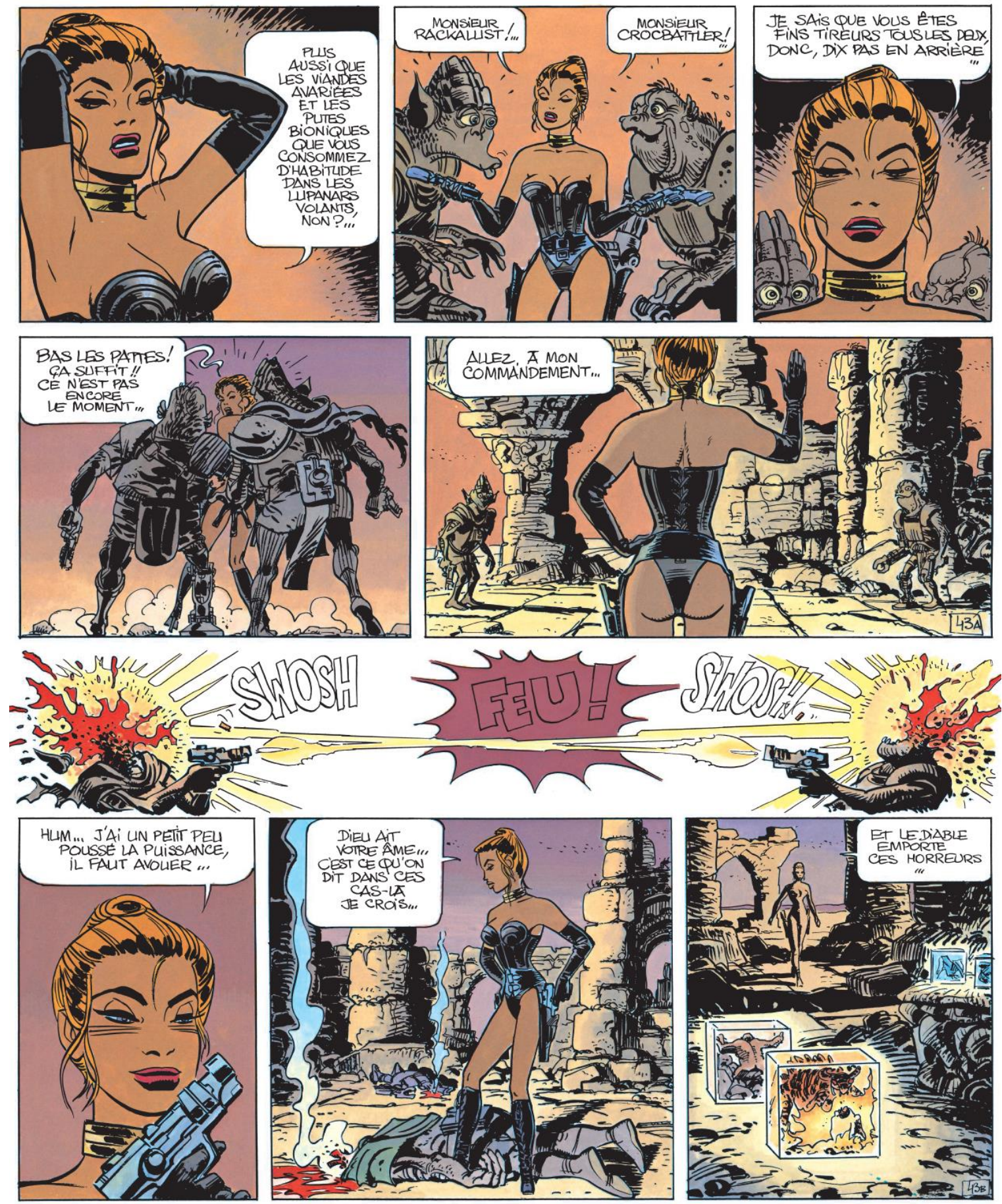

Figure 6- Exemple de meurtre. Laureline fait s'entretuer deux «monstres », don't le statut inférieur s'exprime, entre autres, par le fait qu'ils ne s'expriment pas au moyen de bulles. Valérian - Intégrale nouvelle édition - volume $4:$ p. 113. Christin, Mézières (C) DARGAUD, 2017 

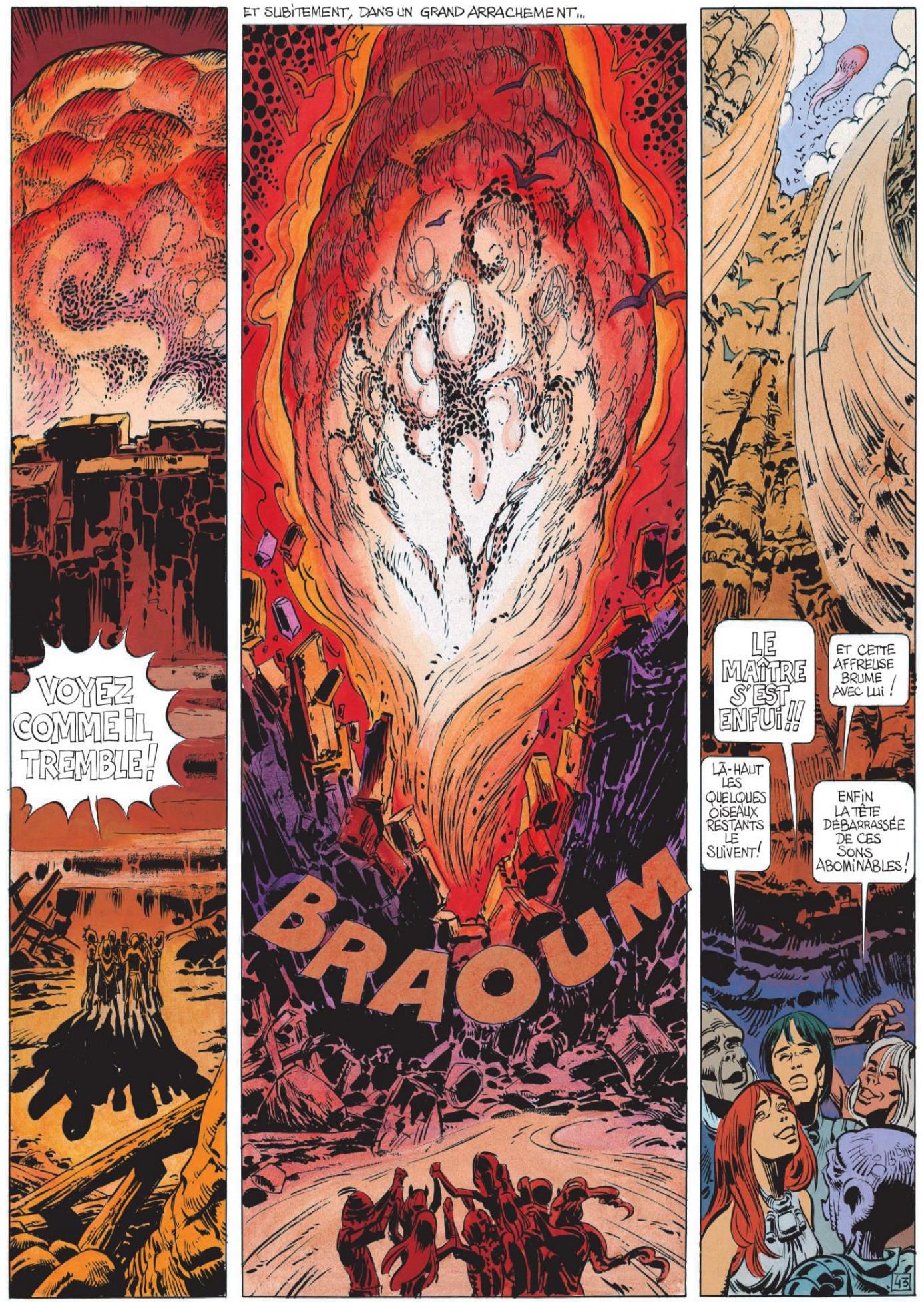

Figure 7 - Exemple de destitution. Finalement, c'est en unissant leurs efforts et leurs pensées que le petit groupe de résistants parvient à chasser le «maître ». Valérian - Intégrale nouvelle édition - volume 2 : p. 167. Christin, Mézières (C) DARGAUD, 2017 
Figure 8 - Exemple de troc. Laureline échange des perles produites par son transmuteur grognon de Bluxte contre une carte de la planète artificielle Point Central. Le troc se pose aux antipodes de l'usage de la violence, puisque tous les participants conservent leur statut de

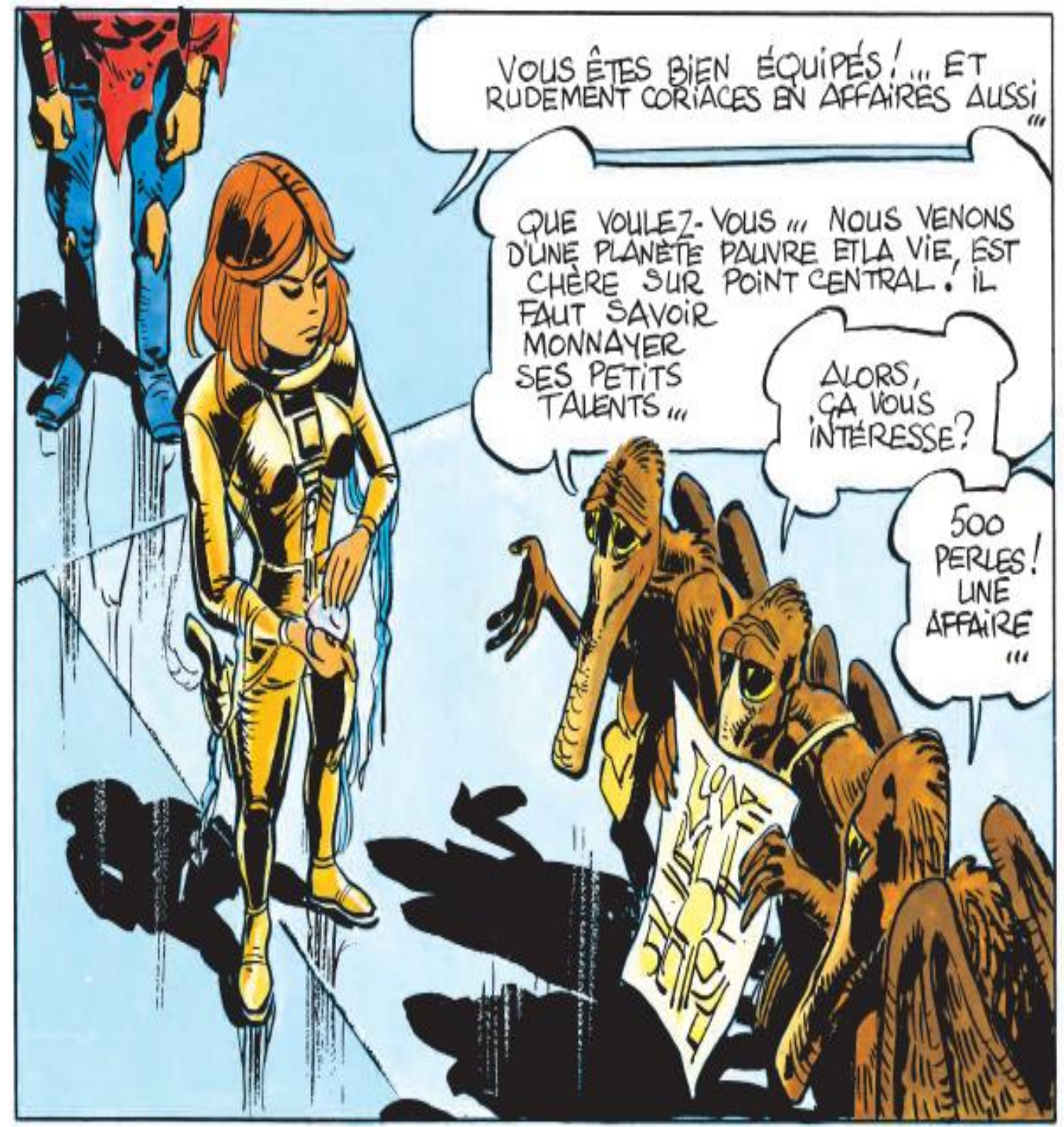

«personne ».

Valérian -

Intégrale

nouvelle

édition -

volume 3 :

case 7, p. 41,

Christin,

Mézières (C)

DARGAUD,

2017 